\title{
Editorial
}

\section{What research topic is the "hot" research topic?}

In the previous editorial (1), a key question related to the decision on selecting the journal for submitting a manuscript is open: "To submit articles for academic advancement or to contribute to evidence pool and education?". I want to contribute to this discussion by commenting on another related aspect.

Another question I hear frequently from students, junior researchers and clinicians, is related to the selection of the research field for their future research career: "What field of science is THE FIELD for an exciting research, for being a successful and recognized researcher and for contributing to innovations?" And a related question: "Is it possible to do research without new sophisticated equipment?", or in other words: "Is it possible to do a good research when I do not have latest (expensive) technology or I am not in the new emerging promising field of science"? So are these two attributes: the latest (expensive) technology in combination with the new emerging promising direction of research the best and required conditions?

The possibilities of research to contribute to evidence pool and education are not limited only to fashionable promising directions and the latest expensive technology. There are many other possibilities of the utmost importance: to reduce gaps in knowledge, to address and benefit from conflicting results, to categorize previous knowledge and findings, to reevaluate the validity of knowledge in the context of current knowledge, or to update the knowledge in the context of current knowledge.

I want to demonstrate it using electrocardiology (ECG) as an example. Electrocardiology is an old science with the very beginning at the end of the 19th century, the clinical ECG at the beginning of the 20th century. Over the years, the diagnostic criteria have been agreed to the extent that they are used for computerized diagnosis, the automated diagnosis being an integral standard part of majority of electrocardiographs - can anything be yet researched?
The definite answer is: "Yes". Just two examples among many - updating the knowledge in the context of current knowledge that extends the classical diagnostic portfolio of ECG evaluation: Brugada syndrome (2) or more recently Bayes syndrome (3).

Brugada syndrome is a rare cardiac arrhythmia associated with ventricular fibrillation and a high risk for sudden cardiac death, predominantly in younger males with structurally normal hearts. Its identification has been associated with major progress in genetics and physiology. Over the past 20 years, extensive research in this field has produced major progress toward better understanding of Brugada syndrome and the gaining of knowledge of the genetic background, pathophysiology and patient management (4).

Another example is the Bayes syndrome, summarizing the complex view on the topic of inter-atrial block (IAB) due to conduction impairment in Bachmann's bundle, first described by Bayes de Luna 30 years ago (5). Accumulated evidence covering most aspects of the pathophysiology, the ECG and vectorcardiographic descriptions, and the association with supraventricular tachyarrhythmias has qualified the advanced IAB for a clinical syndrome accepted by the scientific community $(6,7)$.

These both examples, represent new ECG areas of research - but what about seemingly "established" ECG topics? A good example is the ECG diagnosis of left ventricular hypertrophy (LVH) characteristic for years by conflicting results.

LVH detected either by echocardiography (or other imaging methods) or ECG is a recognized cardiovascular risk factor and the sign of the target organ damage in hypertensive patients, therefore a considerable effort is devoted to its diagnosis. A persisting problem, however, is the low agreement between results of the imaging methods and ECG that is reflected in the low sensitivity of ECG results (8). This has undermined the interest of cardiologists in ECG with respect to LVH and has directed the focus on the imaging methods.

Address for correspondence: Ljuba Bacharova, International Laser Center, Ilkovicova 3, 84102 Bratislava, Slovak Republic.E-mail: bacharova@ilc.sk

Received: 19.01.2018 Accepted: 19.01.2018 Copyright 2018 Heart-Vessels and Transplantation doi: 
This approach is partly understandable: LVH is defined as an increase in the size of an organ or its parts; therefore, the effort to estimate the increased left ventricular (LV) size is logical. The imaging methods can measure the size of individual parts of the heart noninvasively, the estimation of left ventricular mass (LVM) is therefore quite accurate, and these methods are preferred.

However, in the case of ECG this logic is questionable. The ECG diagnosis of LVH is primarily based on so-called voltage criteria - the increased voltage of QRS complex. The increased QRS voltage is observed in a proportion of patients with LVH: based on these findings a simple association has been made - the bigger the LV the higher the QRS voltage. In electro-pathophysiological terms: the bigger the activation front the higher the QRS voltage.

The role of ECG in LVH diagnosis has its historical roots. At the beginning of the last century, cardiologists had only limited possibilities for non-invasive estimation of LVM. ECG was one of a few available non-invasive methods, and the high specificity of the increased QRS voltage in LVH patients allowed considering it as a good basis for confirming the diagnosis. Over the years, there was a continuous effort to improve the ECG-LVH criteria with a common aim - to estimate the increased LVM the best and ECG has been thus used as a surrogate for estimating the increased LV mass. However, in spite of a quite impressive number of recommended ECG criteria for LVH (9), it has been a vain effort. The ECG-LVH diagnostic continues being open and challenged issue (10).

Is there any problem in this logic? Can this problem be addressed differently? Can this problem be challenged? Can it be even turned to a "hot topic"? Let us summarize "what is known" on ECG diagnosis of left ventricular hypertrophy.

First, there is no statistically significant or there is only low correlation between the QRS voltage and LVM. Consequently, if there is no correlation, there is no logic to use QRS voltage for predicting LVM. Thus, there is an essential logical inconsistency: on the one hand, ECG criteria have to be used for predicting/ estimating LVM, on the other hand there is no correlation between the ECG and LVM.
Second, by principle, ECG does not measure the size or mass of the heart. However, and it is of utmost importance, ECG provides a unique information on the electrical characteristics of the heart that is not provided by any other diagnostic methods. Therefore, the key problematic point is the effort to estimate the size/ LVM by a method that measures electrical potentials, namely, to estimate the size of the generator of the cardiac electric field (the heart) by measuring the distribution of electrical potential on the body surface.

Current terminology assigned the discrepancies between ECG and imaging methods showing the increased LVM as false results, either false negative or false positive, and "false" implies "wrong". However, using these terms is misleading. ECG is an objective diagnostic method providing fundamentally different and unique information - and its results cannot be just neglected by assigning them as "false". Differences in LVM as estimated by imaging methods versus QRS voltages and other electrical phenomena indicate different manifestations of underlying electrical versus anatomical remodeling processes. The variety of QRS and T-wave changes that occur in the context of electrically remodeled myocardium reflect the added diagnostic and prognostic value of ECG that needs to be decoded.

The ECG-LVH diagnostic criteria are based on the evidence of the increased QRS voltage. However, the increased QRS voltage has been observed only in the minor part of LVH patients, what is reflected in their low sensitivity (8). Therefore, the effect of other factors leading to the increased QRS voltage needs to be considered. The results of recent clinical, epidemiological and computer simulation studies provide already supporting evidence (11-15).

Since ECG is a method recording the cardiac electric field, future ECG research must be focused on identifying characteristics of electrical phenomena associated with left ventricular hypertrophy. Thus we can better understand their relation to risk for ventricular arrhythmias or to heart failure, i.e., conditions of potentially greater clinical importance than anatomical LVH per se (16). 
By using new technologies, we are able to image electrical processes in the heart and simulate electrical processes to understand them better. It opens endless spectrum of exciting topics for research. Nevertheless, to be very clear, the "hot" topic is not necessarily conditioned by expensive equipment or a fashionable research direction. Firstly, it needs to address and solve important problem. And ECG-LVH is really an exciting long-lasting problem. Traditionally, the dominant arguments in favor of ECG-LVH mentioned frequently are availability and low cost. Yes, they are advantages that allow providing research even with limited resource. However, the main argument in favor for using ECG is its unique information on the electrical characteristics of the heart that we need to decode and understand.

Being involved in the research in electrocardiology for years and seeing the development in science and technology in general and in electrocardiology particular I dare to say that we are living in an exciting period. The research can contribute to creating new diagnostic categories (as e.g. Brugada syndrome and Bayes syndrome), or, what is equally exciting, to lead to re-evaluating old diagnostic paradigms (demonstrated by using ECG diagnosis of LVH as an example).

And the answer to the questions of young scientists? These were just a few examples of many possibilities for research. The clinical electrocardiography is as a top of the iceberg seen above the water. It is worth to go deeper to discover what is underneath. There are millions of ECGs recorded in the world waiting for your analysis and invention. Open your eyes, open your mind, and share your results, contribute to the pool of evidence and knowledge. Nota bene: sharing your ideas and results - i.e. publishing - this is the mission of any research.

Ljuba Bacharova

International Laser Center, Bratislava, Slovak Republic
Institute of Pathophysiology, Medical Faculty of Comenius University, Bratislava, Slovak Republic

Peer-review: Internal

Conflict of Interest: None to declare

Authorship: L.B.

Acknowledgement and funding: None to declare

\section{References}

1.Kudaiberdieva G. A short message from the Editor-InChief: submit articles for academic advancement or contribute to evidence pool and education. Heart Vessels and Transplantation 2017;1: 37-8.

2.Brugada R, Campuzano O, Sarquella-Brugada G, Brugada J, Brugada P. Brugada syndrome. Methodist Debakey Cardiovasc J 2014;10: 25-8.

3.Bacharova L, Wagner GS: The time for naming the Interatrial Block syndrome: Bayes Syndrome. J Electrocardiol 2015; 48: 133-4.

4.Veerakul G, Nademanee K. Brugada syndrome: two decades of progress. Circ J 2012; 76: 2713-22.

5.Bayes de Luna A1, Cladellas M, Oter R, Torner P, Guindo J, Marti V, et al.: Interatrial conduction block and retrograde activation of the left atrium and paroxysmal supraventricular tachyarrhythmia. European Hear J 1988; 9: 1112-8.

6.Bayes de Luna A, Platonov P, Garcia-Cosio F, Cygankiewicz I, Pastore C, Baranowski R, et al. Interatrial blocks: a separate entity from left atrial enlargement: a consensus report. J Electrocardiol 2012; 45: 445- 51.

7.Conde D, Baranchuk A, Bayés de Luna A: Advanced interatrial block as a substrate of supraventricular tachyarrhythmias: a well-recognized syndrome. J Electrocardiol 2015; 48: 135-40.

8.Pewsner D, Juni P, Egger M, Battaglia M, Sundstrom J, Bachmann LM. Accuracy of electrocardiography in diagnosis of left ventricular hypertrophy in arterial hypertension: systematic review. BMJ 2007; 335: 711. 
Heart, Vessels and Transplantation 2018; 2: doi

On research topics

Bacharova

9. Hancock EW, Deal BJ, Mirvis DM, Okin P, Kligfield P, Gettes LS, et al. American Heart Association Electrocardiography and Arrhythmias Committee, Council on Clinical Cardiology; American College of Cardiology Foundation; Heart Rhythm Society. AHA/ACCF/HRS recommendations for the standardization and interpretation of the electrocardiogram: part $\mathrm{V}$ : electrocardiogram changes associated with cardiac chamber hypertrophy: a scientific statement from the American Heart Association Electrocardiography and Arrhythmias Committee, Council on Clinical Cardiology; the American College of Cardiology Foundation; and the Heart Rhythm Society. Endorsed by the International Society for Computerized Electrocardiology. J Am Coll Cardiol 2009; 53: 992-1002.

10.Bacharova L. What is recommended and what remains open in the American Heart Association recommendations for the standardization and interpretation of the electrocardiogram. Part V: electrocardiogram changes associated with cardiac chamber hypertrophy. J Electrocardiol 2009;42: 388-91. 11.Bacharova L, Szathmary V, Kovalcik M, Mateasik A. Effect of changes in left ventricular anatomy and conduction velocity on the QRS voltage and morphology in left ventricular hypertrophy: a model study. J Electrocardiol 2010; 43: 200-8.

12.Svehlikova J, Zelinka J, Bacharova L, Tysler M. Modeling and visualization of the activation wavefront propagation to improve understanding the QRS complex changes indicating left ventricular hypertrophy. J Electrocardiol 2016; 49: 755-62.

13.Bacharova L, Chen H, Estes EH, Mateasik A, Bluemke $D$, Lima J, et al. Determinants of discrepancies in detection and comparison of the prognostic significance of left ventricular hypertrophy by electrocardiogram and cardiac magnetic resonance imaging. Am J Cardiol 2015; 115: 515-22.

14.Estes EH, Zhang Z-M, Li Y, Tereshchenko LG, Soliman EZ. The Romhilt-Estes left ventricular hypertrophy score and its components predict all-cause mortality in the general population. Am Heart J 2015; 170: 104-9.

15.Estes EH, Zhang Z-M, Li Y, Tereshchenko LG, Soliman EZ. Individual components of the Romhilt-Estes left ventricular hypertrophy score differ in their prediction of cardiovascular events: the Atherosclerosis Risk in Communities (ARIC) Study. Am Heart J 2015; 170: 12206.

16.Bacharova L, Estes HE, Schocken DD, Ugander $M$, Soliman EZ, Hill JA, et al. The 4th report of the working group on ECG diagnosis of left ventricular hypertrophy. J Electrocardiol 2017; 50: 11-5. 